\title{
Reduced Brain-Derived Neurotrophic Factor is associated with childhood trauma experiences and number of depressive episodes in severe mental disorders
}

\begin{abstract}
Monica Aas ${ }^{1 *}$, Ingrid Dieset ${ }^{1}$, Ragni Mørch ${ }^{1}$, Nils Eiel Steen ${ }^{1}$, Sigrun Hope ${ }^{1}$, Elina J. Reponen ${ }^{1}$, Jannicke F. Laskemoen ${ }^{1}$, Thor Ueland ${ }^{2-4,5}$, Pål Aukrust ${ }^{2-4}$, Ingrid Melle ${ }^{1}$, Ingrid Agartz ${ }^{1,6,7}$, Ole A. Andreassen ${ }^{1}$
\end{abstract}

${ }^{1}$ NORMENT K.G Jebsen Centre for Psychosis Research, Institute of Clinical Medicine, Norway;

${ }^{2}$ Research Institute of Internal Medicine, Oslo University Hospital Rikshospitalet, Oslo, Norway;

${ }^{3}$ Section of Clinical Immunology and Infectious Diseases, Oslo University Hospital, Rikshospitalet, Norway;

${ }^{4}$ K.G. Jebsen Inflammatory Research Center, University of Oslo, Oslo, Norway;

${ }^{5}$ K.G. Jebsen - Thrombosis Research and Expertise Center (TREC), University of Tromsø, Tromsø, Norway;

${ }^{6}$ Department of Psychiatric Research, Diakonhjemmet Hospital, Oslo, Norway, and

${ }^{7}$ Department of Clinical Neuroscience, Karolinska Institutet, Stockholm, Sweden

*Corresponding Author:

Monica Aas, PhD

NORMENT KG Jebsen Psychosis Research Centre

Division of Mental Health and Addiction, Institute of Clinical Medicine

Bygg 49, Ullevål sykehus,

PO Box 4956 Nydalen, 0424 Oslo, Norway

Email: monica.aas@medisin.uio.no

Key words: BDNF, severe mental disorders, childhood trauma, number of depressive episodes. 


\begin{abstract}
Background: Although several studies have found reduced plasma BDNF levels in patients with severe mental disorders, the sample sizes have been small and have exhibited variation and heterogeneity. Furthermore, long-term neurobiological changes following childhood trauma and clinical severity have been linked to a reduction in BDNF levels. Accordingly, we aim to clarify, using the largest sample size to date, the role of plasma BDNF in individuals with severe mental disorders in relation to the number of episodes, current remission status, and childhood trauma experiences.
\end{abstract}

Methods: The study sample comprised 1446 individuals (schizophrenia: SZ [n=589]; bipolar disorder: BD [n=254]); and healthy control: HC [n=603]) all recruited from the same catchment area. A subsample ( $\mathrm{N}=629)$ of this larger group had a history of childhood trauma, and some ( $\mathrm{N}=195)$ participated in a one-year follow-up study. The level of BDNF in plasma was measured, and childhood trauma was assessed using the Childhood Trauma Questionnaire (CTQ). Diagnoses and episodes were obtained using the Structured Clinical Interview (SCID).

Results: Patients with SZ or BD had lower levels of plasma BDNF than did the HC group ( $\mathrm{p}=0.002, \mathrm{p}=0.003$, respectively). Within patients, reduced plasma BDNF levels were associated with more depressive episodes $(\mathrm{p}=0.04)$. Longer time in remission after depressive episodes was associated with higher plasma BDNF levels ( $\mathrm{p}=0.02$ ), and patients reporting childhood sexual abuse exhibited lower plasma BDNF levels $(p=0.049)$ than patients without sexual abuse.

Conclusion: Our study confirms that patients with a severe mental disorder exhibit reduced BDNF levels. While the strongest reduction in BDNF was observed in patients reporting childhood sexual abuse, reduced BDNF levels were also associated with more depressive episodes. Accordingly, further studies are warranted to determine whether treatment that increases BDNF levels may be beneficial to these individuals. 


\section{INTRODUCTION}

Schizophrenia spectrum disorder (SZ) and bipolar spectrum disorder (BD) affect 2 to 3\% of the population and cause marked decrements in quality of life and life expectancy (Whiteford et al., 2013). The core characteristic of SZ is psychotic symptoms, whereas affective symptoms are at the core of BD. There is, however, an overlap in these symptoms, as described in the continuum hypotheses of the two disorders (Craddock and Owen, 2010). SZ and BD are highly heritable (Lichtenstein et al., 2009) and involve complex genetic and environmental interactions (Burmeister et al., 2008). Furthermore, childhood trauma, drug addiction and poor lifestyle are environmental factors associated with both of these disorders (Aas et al., 2016; Di Forti et al., 2015; Varese et al., 2012). Thus, because a substantial number of patients do not respond sufficiently to treatment (Mcgorry et al., 2014, Scott et al., 2013), it is urgent to find relevant biomarkers and personalized treatment targets.

Brain-derived neurotrophic factor (BDNF) is essential for the human brain. It is a protein produced by many cell types, including neurons and immune cells, and its levels are influenced by mental stress, exercise and diet (Firth et al., 2015, Solati et al., 2015). BDNF promotes neuronal growth and differentiation during brain development and increases synaptic plasticity and the maintenance of neurons in adult life (Nuernberg et al., 2016). Furthermore, BDNF crosses the blood-brain barrier (BBB) (Fernandes et al., 2015a), and its peripheral levels are highly correlated with its levels in the cerebrospinal fluid (r=0.8) (Fernandes et al., 2015a). Over the last decade, numerous studies have found low levels of peripheral BDNF in individuals with neurodegenerative and neuropsychiatric disorders (Nuernberg et al., 2016). Low levels of peripheral BDNF are observed in patients with both affective and non-affective severe mental disorders, including major depressive disorder (MDD) (Bus et al., 2011), BD (Fernandes et al., 2015b) and SZ (Fernandes et al., 2015a). 
To this end, mechanisms driving reductions in peripheral BDNF levels in individuals with these disorders are unclear. Later clinical illness course characterized by more episodes (Kauer-Sant'Anna et al., 2009, Fernandes et al., 2015a, Fernandes et al., 2015b, Nuernberg et al., 2016) and/or by being in a current mood or psychotic episode (Bus et al., 2015, Nuernberg et al., 2016) has been suggested to influence BDNF levels. BDNF has also been suggested as a marker of the stage of the illness (Fernandes et al., 2015a, Fernandes et al., 2015b, Nuernberg et al., 2016) whereby a lower BDNF level indicates the patient is in a later stage of the illness. Indeed, a recent meta-analysis of BD confirms that peripheral BDNF levels are reduced to the same extent within a manic or depressive episode but that they are not reduced not during a euthymic state (Fernandes et al., 2015b). Thus, the temporal relationship between peripheral plasma and serum BDNF levels and symptoms must be clarified. Bus et al., (2015) recently determined that patients with a treatment-resistant depression were more likely to have lower serum BDNF levels over a two-year period, whereas patients with higher serum BDNF levels were more likely to be in remission at follow-up. Hence, taking into consideration remission status when investigating peripheral BDNF levels and illness staging is warranted, especially given that these relationships are, at present, unclear in SZ and BD.

In addition, interactions between high exposure to childhood trauma experiences and peripheral BDNF levels in plasma have been proposed (Aas et al., 2014b, Mondelli et al., 2011). Childhood trauma is an influential risk factor for developing a wide range of mental illnesses, including psychotic disorders (Varese et al., 2012) and is also associated with a more severe disease course (Aas et al., 2016, Yung et al., 2015). BDNF protects the brain from the toxic effects of glucocorticoids (stress hormone) and supports neurogenesis (Taliaz et al., 2010). Glucocorticoids, on the other hand, suppress BDNF mRNA in the CA 3 region 
of the hippocampus and reduce dendrite density accordingly (Bennett \& Lagopoulos, 2014. )Although it has been previously concluded that experiences of severe stress, such as childhood traumas, reduce BDNF plasma levels (Mondelli et al., 2011; Aas et al., 2014b), it is possible that reduced BDNF plasma levels prior to childhood trauma worsen the adverse effects of childhood trauma experiences on brain development and promote more severe neurodevelopmental changes following trauma experiences. Thus, the influence of childhood trauma on BDNF within the psychosis continuum disorder and its association with illness must be further explored.

Peripheral BDNF has been frequently studied in BD and SZ; however, the sample sizes in these studies have been small, i.e., less than 200, and recent meta-analyses may have been hampered by the variations in the BDNF measurement methodology and the heterogeneity among the populations included in the studies (Nuernberg et al., 2016). To address these issues, we investigated peripheral BDNF levels measured in plasma among a large group of individuals ( $\mathrm{N}=1446)$ that consisted of patients with severe mental disorders, i.e., SZ or BD, and an HC group from the same catchment area. Specifically, we aimed to i) replicate reduced plasma BDNF levels in patients with psychosis continuum disorder compared to the HC group while correcting for childhood trauma experiences; ii) investigate whether plasma BDNF levels are associated with more affective and psychotic episodes as a marker of a more prolonged clinical staging during longitudinal testing; and iii) rule out that current medications, namely, antipsychotics or antidepressants are influencing BDNF. 


\section{MATERIALS AND METHODS}

\section{Participants}

The participants were recruited consecutively from psychiatric units, both outpatient and inpatient, of 4 major hospitals in Oslo as part of the Thematically Organized Psychosis (TOP) study. All participants were recruited from the same catchment area that is composed of individuals of similar socio-economic status (SES) and ethnic backgrounds (88\% Caucasians). A total of 1446 participants (individuals with schizophrenia [ $\mathrm{n}=589$ ], individuals with bipolar disorder [ $n=254]$ ), and healthy individuals [n=603]) were recruited. A subset of individuals provided data regarding childhood trauma experiences, specifically, $\mathrm{N}=629$ (SZ: N=263; BD: N=132; HC: N=234). In addition, data were collected from 195 individuals provided one year after the initial study ( $N=195 ; S Z, N=51 ; B D, N=50 ; H C$, $\mathrm{N}=94$ ). Inclusion criteria for the clinical groups included age range between 18 and 65 years and a DSM diagnosis of schizophrenia spectrum disorder (SZ) or bipolar spectrum disorder (BD). Inclusion criteria for the healthy controls were included the following: live in the same district as the patients, beg between 18 and 65 years of age and have no diagnosis during their lifetime of a DSM1 disorder. Exclusion criteria for all groups included the following: organic psychosis, neurological disorder, unstable or uncontrolled medical conditions that interfere with brain function, and age outside the range of 18 to 65 years. The Regional Committee for Medical Research Ethics and the Norwegian Data Inspectorate approved the study. All participants provided written informed consent and were enrolled in the study between 2007 and 2016.

\section{Clinical Assessment}

Trained physicians, psychiatrists, and clinical psychologists performed clinical assessments. Diagnoses were based on the Structured Clinical Interview for DSM-IV Axis I disorders 
(SCID-I), chapters A-E. All clinical personnel completed a training program in diagnostics and symptom rating that was based on the training program at UCLA (Los Angeles, California). The training program is based on the SCID 101 training videos and includes videos with reliability testing between SCID scorers (http://www.scid4.org/index.html). The diagnostic reliability was found to be satisfactory with an overall agreement regarding the DSM-IV diagnostic categories of $82 \%$ and an overall $\kappa$ of 0.77 (95\% CI: $0.60-0.94)$.

\section{Clinical characteristics}

Remission was measured in weeks. Remission of psychotic symptoms was defined as PANSS items below four for the following items: P1, P3, G9, P2, G5, N1, N4, and N6. Remission was based on Nancy Andreasen’s six-month criteria (Andreasen, et al., 2015). Remission of affective episodes was based on the SCID definitions defined as symptoms during the last two months. The illness stage was measured by numbers of episodes and was calculated based on the Diagnostic and Statistical Manual of Mental Disorders (DSM) verified episode criteria using the Structured Clinical Interview (SCID). According to these criteria, the following data were reported: $\mathrm{N}=513$ (61\%) had at least one depressive episode; $\mathrm{N}=665$ (79\%) had at least one psychotic episode; and N=201 (24\%) had at least one manic episode. Within the SZ group, the distributions of depressive, psychotic and manic episodes (mean $\pm \mathrm{SD}$ ) were $2.77 \pm 10.18,4.18 \pm 14.58$, and $0.26 \pm 1.41$, respectively. Within the BD group, the distributions of depressive, psychotic and manic episodes (mean \pm SD) were $6.23 \pm 9.98,1.33 \pm 2.63$, and 2.07 \pm 6.78 , respectively. Moreover, $45 \%$ of the patients had at least two types of episodes and 15\% had three types of episodes.

Clinical illness stage modeling in psychiatry has adopted the numerical system that is used in medical staging models (Berk et al., 2017, McGorry et al., 2014, Scott et al., 2013). The 
advantage of this model is that it explicitly considers the evolution of psychopathology during the development of a mental illness and emphasizes that the progression of the illness is by no means inevitable but rather that it can be altered by providing appropriate interventions that target individual modifiable risk and protective factors (McGorry et al., 2014). Stage progression is usually defined in four steps, namely, stage 0 is an asymptomatic stage or atrisk or latency phase. Stage 1 is often called the prodromal phase and consists of stage 1A, which is a nonspecific distress period, and stage $1 \mathrm{~B}$, which is a subthreshold high-risk stage. Stage 2 is the first-illness episode stage. Stage 3 is characterized by single or multiple recurrences of illness episodes and consists of stage 3A, which is characterized by a recurrence/persistence of episodes; stage 3B, which is a first threshold relapse phase; and stage 3C, which is a stage noted by multiple relapses. Finally, stage 4 is a treatment resistance or chronic disease stage (Berk et al., 2017, McGorry et al., 2014). In the current study, the number of episodes was used as a measure of illness stage.

\section{BDNF measurement}

For the BDNF analysis, blood was drawn into EDTA-containing vials, and plasma was extracted and stored at $-80{ }^{\circ} \mathrm{C}$. BDNF levels were measured using enzyme immune-assay with antibodies from R\&D systems (Stillwater, MN). Intra-and inter-assay coefficients of variance were less than $10 \%$.

\section{Childhood trauma}

To measure childhood adverse events, we used the Childhood Trauma Questionnaire (CTQ), a retrospective questionnaire that assesses traumatic experiences in childhood. The CTQ has answers ranging from never true, rarely true, sometimes true, often true, to very true, and it yields a total score as well as five subscores, namely, physical, emotional and sexual abuse 
and physical and emotional neglect (Bernstein et al., 1994). For this study, we focused on the most severe form and most studied subtype of childhood trauma, sexual abuse (Nemeroff 2016). Thus, the main analysis was performed only for sexual abuse. In addition, a total trauma score was also calculated. Data were analyzed and divided into below or above the moderate to severe cutoff scores, as suggested by Bernstein et al., (1994). The reliability and validity of the CTQ have been demonstrated previously (Aas et al., 2014b, Bernstein et al., 1994).

\section{Statistics}

Data were analyzed using Predictive Analytic software, SPSS, Version 22 (IBM Corp., USA), and continuous variables are presented as the mean \pm SD. For descriptive analyses (Tables 1 ), we used chi-square tests and univariate ANOVAs.

Linear multiple regression analyses were performed to investigate the numbers of episodes and were divided into depressive, manic, and psychotic episodes. To test whether being in remission was associated with an increase in plasma BDNF levels (Berk et al., 2011), linear regression analyses were performed to determine whether the length (in weeks) of remission of affective (depressive or manic) or psychotic episodes was linked to an increase in plasma BDNF levels. Data were available for a subset of individuals for a year follow-up analysis (N=195; SZ: N=51; BD: N=50: healthy controls: N=94). Plasma BDNF levels at baseline, plasma BDNF levels at one-year follow-up and changes in plasma BDNF levels from baseline to the one-year follow-up, baseline minus follow-up, were compared to remission status at the one-year follow-up. 
Gender differences in BDNF signaling and functions are reported, and estrogens are found to have a potential positive regulatory effect on BDNF expression and signaling (Chan \& Ye, 2017). It has been suggested that gender differences exist in the relationship between childhood trauma and the clinical expression of patients with psychotic disorders and that women experience greater negative effects than men (Fisher et al., 2009; Garcia et al., 2016; Haug et al., 2014). Therefore, analyses investigating BDNF levels and reports of trauma, as well as analyses of the potential role of childhood trauma on BDNF levels, were performed separately on females and males before they were analyzed together as one group. In addition to gender, several other environmental factors have been linked to BDNF levels, including age, cigarette smoking and time of stored samples (Bus et al., 2011). Thus, all of these environmental factors were taken into account in the current study. To test staging based on the numbers of episodes and not on the length of the illness, age at first episode and current age were added into the multivariate model as independent variables.

As current smoking status was available for the patient group but not for the healthy control group, smoking was corrected for only within the patient sample.

To rule out medication as an influencing factor, current medications were included in the analyses and were accordingly divided into no current medication use, one type of antipsychotic medication use, two or more types of antipsychotic medication use, and antidepressant medication use (yes/no). The threshold for statistical significance was set at $\mathrm{p} \leq 0.05$ with post hoc Bonferroni corrections. 


\section{RESULTS}

\section{Sample characteristics}

The socio-demographic and clinical characteristics of the total sample $(\mathrm{N}=1446)$, including the diagnostic subgroups (BD, SZ) and healthy controls are presented in Table 1. All participants were recruited from a catchment area in Norway. There were more males in the SZ group and more females in the BD group. There were more Caucasians in the healthy control group, $96 \%$, compared to the patient group $\leq 71 \%$. Patients with an SZ disorder had more psychotic episodes, while the BD group had more manic and depressive episodes. Controls had lower childhood trauma scores based on the CTQ than did both the SZ and BD groups (see Supplementary Material Table S1 for total CTQ scores and subtypes of trauma). Post hoc Bonferroni analyses revealed higher physical neglect scores among those with SZ compared to those with BD. Otherwise, there were no statistically significant differences in trauma scores between the two patient groups.

-Please insert Table 1 around here-

\section{BDNF levels in relation to case-control status and number of episodes}

Plasma BDNF levels were higher among healthy controls than it was for patients with BD and $\mathrm{SZ}, \mathrm{p}=0.003, \mathrm{p}=0.002$, respectively (see Figure 1 ). Post hoc differences were nonsignificant for patient comparisons, i.e., between patients with BD and patients with SZ. A within-patient analysis found there were no differences in BDNF levels between females and males (mean \pm SD: 5.82 $\pm 3.76,5.91 \pm 3.45$, respectively, $\mathrm{t}=0.36 \mathrm{P}=0.71$ ). However, among healthy 
individuals, a trend was observed for higher BDNF levels in females compared to males (mean \pm SD, $6.87 \pm 3.63,6.34 \pm 3.54$, respectively, $\mathrm{t}=-1.84 \mathrm{P}=0.067$ ).

The number of depressive episodes was associated with lower plasma BDNF levels in the population as a whole $(\Omega=-0.07, \mathrm{t}=-2.07, \mathrm{p}=0.04)$. These findings were independent of time since sampling (in years), patient status, current age, gender, age at onset and daily smoking (yes/no) (Table 2). These findings remained significant when the state of current symptoms, i.e., in remission or not, was added into the model ( $ß=-0.08, \mathrm{t}=-2.41, \mathrm{p}=0.02)$. The relationship between plasma BDNF level and the number of psychotic or manic episodes was not significant $(\mathrm{p}>0.1)$. Dividing into females and men, findings were similar for both females and men, however, the association was no longer statistical significant for either groups (data not shown).

-Please insert Figure 1, Table 2 around here-

\section{BDNF levels and one-year remission status}

Longer time in remission from a depressive episode was associated with higher plasma BDNF levels ( $ß=0.19, t=2.32, p=0.02$, Figure 2 ). No relationship was observed regarding the length of remission from a psychotic or manic episode and plasma BDNF levels ( $p>0.1$ ), and no associations were observed between plasma BDNF levels at baseline or plasma BDNF at follow-up and a classification of remitted at the one-year follow-up ( $\mathrm{p}>0.1$, see Supplementary Material, Table S2) or between current antipsychotic or depressive medication and plasma BDNF levels at baseline or follow-up ( $p>0.1)$. Twenty-five $\%$ of the patients were taking both antidepressants and antipsychotic medication at baseline. 


\section{BDNF levels and childhood trauma}

In the total sample, a dose-relationship was observed between more total childhood trauma experiences and lower BDNF levels ( $\mathrm{f}=3.09, \mathrm{p}=0.02$; see Supplementary Material Figure $\mathrm{S} 1$ ). Moreover, childhood trauma was associated with reduced BDNF levels in both females and males, with the highest rho occurring in the female patients (see Table 3).

Patients reported more frequent childhood sexual abuse experiences compared to the HCs, with $21 \%$ of the patients reporting sexual abuse experiences that reach levels of moderate to severe cut-off scores by Bernstein et al. (1994) compared to 3\% among the HC group. With respect to the patients, childhood sexual abuse was associated with a reduction in plasma BDNF levels ( $\mathrm{f}=3.08, \mathrm{p}=0.049$, see Supplementary Material Figure S2) and more psychotic and affective episodes (see Supplementary Material Figure S3 and Figure S4). Both females and males who reported childhood trauma experiences exhibited more episodes $(\mathrm{p}<0.05)$. Even after correcting for childhood trauma experiences, patients still reported lower plasma BDNF levels than did the HC group $(\mathrm{f}=19.5, \mathrm{p}<0.001)$.

-Please insert Table 3 around here- 


\section{DISCUSSION}

In this large population of patients with bipolar disorder (BD) or schizophrenia spectrum disorder (SZ), peripheral plasma BDNF levels were decreased compared to the healthy control (HC) group. Low peripheral plasma BDNF levels were associated with more depressive episodes, thus indicating a more severe illness (Mcgorry et al., 2014). Our data from this large study support reduced BDNF levels as a potential biomarker for clinical staging in patients with severe mental disorders, as suggested by previous smaller studies (Berk et al., 2017, Fernandes et al., 2015a, Fernandes et al., 2015b, Nuernberg et al., 2016). Furthermore, we observed that more episodes (depressive, but not psychotic, or manic) were associated with reduced plasma BDNF levels even after correcting for potential confounders. Previous research indicates that more episodes are linked to treatment resistance, cognitive impairments (Aas et al., 2014, Berk et al., 2017, Ortiz et al., 2017) and reduced hippocampal volume, all of which are part of the staging model (Berk et al., 2017, Mcgorry et al., 2014). Our study provides new knowledge on the number of episodes and biological mechanisms, including the reduction in plasma BDNF levels.

Our data also indicate that patients currently in remission from a depressive episode demonstrate higher plasma BDNF levels, suggesting that reduced plasma BDNF levels are associated with ongoing disease. Our findings are consistent with a large study of individuals with MDD conducted by Bus et al., (2015). Their study concluded that patients who remained in a depressive episode without responding to treatment over a two-year period exhibited lower peripheral BDNF levels. In our subsample of data collected one year after the initial study, no significant relationship was observed between peripheral plasma BDNF levels and 
remission. However, it is noted that for our one-year-follow-up study, the sample was rather small, specifically, 101 patients and 94 HCs.

Our study determined that the patients exhibited reduced plasma BDNF levels compared to the control group even after correcting for childhood trauma experiences. As a history of childhood trauma was negatively correlated with BDNF levels, our study indicated that childhood trauma resulted in a reduction in BDNF levels combined with a further non-trauma BDNF reduction in the patient group. The research indicates that approximately $10 \%$ or more of the general population reports a history of childhood trauma (Pruessner et al., 2017, Aas et al., 2016). It could be hypothesized that low BDNF levels reduce resilience to childhood trauma during brain development, thus linking trauma to psychopathology. As glucocorticoid also reduces BDNF mRNA levels in the hippocampus, a brain area important for cognition (Bennett \& Lagopoulos, 2014), patients with low plasma BDNF levels who have a history of childhood trauma, i.e., a history of severe stressors, may be more inclined to exhibit a more progressive illness as characterized by more episodes. This hypothesis must be further clarified in future studies. As recently suggested, treatment aimed to increase BDNF levels may have a positive effect among patients with BD and SZ (Berk et al., 2017, Fernandes et al., 2015a, Fernandes et al., 2015b, Nuernberg et al., 2016). For example, 90 minutes of weekly physical exercise may increase BDNF levels and reduce depressive symptoms (Firth et al., 2015). Another potential treatment to increase BDNF levels is food supplements with zinc. Interestingly, zinc increased serum BDNF levels and decreased depressive symptoms in overweight and obese subjects in a double-blind, randomized, placebo-controlled trial (Solati, et al., 2015). 
It has been suggested that females exhibit higher BDNF levels than males (Chan \& Ye, 2017). In our study, however, while a trend was observed for higher plasma BDNF levels in healthy females, with respect to the patients, no differences between females and males were observed for plasma BDNF levels. We also found that irrespective of gender, a history of childhood trauma was significantly associated with a reduction in plasma BDNF levels and more clinical episodes.

The strength of the current study is the large $\mathrm{N}$ and the homogeneity of the sample. That is, the participants were all recruited from the same catchment area, which was composed of individuals with similar socioeconomic backgrounds within a welfare society. The current study has some limitations. The relevance of a staging model for schizophrenia has been questioned (Bora et al., 2017). Furthermore, investigating numbers of episodes as a marker of staging and clinical severity may be biased by disease characteristics. Some individuals may have a more severe and deteriorating presentation and course from onset, while others may have an episodic illness with full inter-episode recovery. Additionally, a linear progression of a serial of phases may not apply to the course of a disease in all patients. It is also unclear whether treatment resistance leads to more episodes or whether more episodes lead to treatment resistance, as suggested in the stage modeling of severe mental disorders (Mcgorry et al., 2014, Scott et al., 2013). Reduced peripheral BDNF levels were also associated more with depressive than with psychotic or manic episodes, indicating that BDNF levels may be related to affective rather than non-affective episodes. Moreover, a history of childhood sexual abuse was collected retrospectively by asking adults about experiences in childhood. As retrospective interviews correlate with longitudinal case notes about trauma history (Widom et al., 2005), they support the use of retrospective interviews with respect to trauma. 
To conclude, our study demonstrates reduced BDNF levels in patients with severe mental disorders. Reduced BDNF was found across patients diagnosed with SZ and BD, with the most substantial reduction in patients reporting childhood sexual abuse. Our findings support an association between reduced peripheral plasma BDNF levels and more affective episodes, i.e., a putative marker of illness severity. Future studies should include not only numbers of episodes but also other factors suggested for clinical staging, including cognitive function, and structural MRI measures. Reduced plasma BDNF levels could be one biomarker of a more severe clinical course, thus suggesting that treatments designed to increase levels of BDNF may be beneficial for these individuals.

\section{FIGURE LEGENDS}

Figure 1: Patients with a psychosis continuum disorder have reduced plasma BDNF levels compared to healthy individuals.

Figure 2: Longer time in remission of an affective episode is associated with higher plasma BDNF levels. 


\section{References:}

Aas, M., Andreassen, O. A., Aminoff, S. R., Faerden, A., Romm, K. L., Nesvag, R., Berg, A. O., Simonsen, C., Agartz, I. \& Melle, I. (2016). A history of childhood trauma is associated with slower improvement rates: Findings from a one-year follow-up study of patients with a first-episode psychosis. BMC. Psychiatry 16, 126.

Aas, M., Dazzan, P., Mondelli, V., Melle, I., Murray, R. M. \& Pariante, C. M. (2014). A systematic review of cognitive function in first-episode psychosis, including a discussion on childhood trauma, stress, and inflammation. Front Psychiatry 4, 182.

Aas, M., Haukvik, U. K., Djurovic, S., Tesli, M., Athanasiu, L., Bjella, T., Hansson, L., Cattaneo, A., Agartz, I., Andreassen, O. A. \& Melle, I. (2014b). Interplay between childhood trauma and BDNF val66met variants on blood BDNF mRNA levels and on hippocampus subfields volumes in schizophrenia spectrum and bipolar disorders. J. Psychiatr. Res. 52, 5762.

Aas, M., Henry, C., Andreassen, O. A., Bellivier, F., Melle, I. \& Etain, B. (2016b). The role of childhood trauma in bipolar disorders. Int J Bipolar Disord 4, 2.

Agius, M., Goh, C., Ulhaq, S., McGorry, P., 2010. The staging model in schizophrenia, and its clinical implications. Psychiatria Danubina 22(2), 211-220.

Bennett, M. R., \& Lagopoulos, J. (2014). Stress and trauma: BDNF control of dendritic-spine formation and regression. Progress in Neurobiololgy, 112, 80-99.

Bora, E., 2017. A neurodevelopment and neuroplasticity-based framework for early intervention in psychotic disorders. Psychological medicine, 1-9.

Berk, M., Brnabic, A., Dodd, S., Kelin, K., Tohen, M., Malhi, G. S., Berk, L., Conus, P. \& McGorry, P. D. (2011). Does stage of illness impact treatment response in bipolar disorder? Empirical treatment data and their implication for the staging model and early intervention. Bipolar Disord 13, 87-98.

Berk, M., Post, R., Ratheesh, A., Gliddon, E., Singh, A., Vieta, E., Carvalho, A. F., Ashton, M. M., Berk, L., Cotton, S. M., McGorry, P. D., Fernandes, B. S., Yatham, L. N. \& Dodd, S. (2017). Staging in bipolar disorder: from theoretical framework to clinical utility. World Psychiatry 16, 236-244.

Bernstein, D. P., Fink, L., Handelsman, L., Foote, J., Lovejoy, M., Wenzel, K., Sapareto, E. \& Ruggiero, J. (1994). Initial reliability and validity of a new retrospective measure of child abuse and neglect. Am. J. Psychiatry 151, 1132-1136.

Bose, R., Moors, M., Tofighi, R., Cascante, A., Hermanson, O. \& Ceccatelli, S. (2010). Glucocorticoids induce long-lasting effects in neural stem cells resulting in senescence-related alterations. Cell Death Dis 1, e92. 
Bureau/ACYF/ACF/HHS, C. s. (2017). https://www.acf.hhs.gov/cb.

Burmeister, M., McInnis, M. G. \& Zollner, S. (2008). Psychiatric genetics: progress amid controversy. Nat Rev Genet 9, 527-40.

Bus, B. A., Molendijk, M. L., Penninx, B. J., Buitelaar, J. K., Kenis, G., Prickaerts, J., Elzinga, B. M. \& Voshaar, R. C. (2011). Determinants of plasma brain-derived neurotrophic factor. Psychoneuroendocrinology 36, 228-239.

Bus, B. A., Molendijk, M. L., Tendolkar, I., Penninx, B. W., Prickaerts, J., Elzinga, B. M. \& Voshaar, R. C. (2015). Chronic depression is associated with a pronounced decrease in plasma brain-derived neurotrophic factor over time. Mol. Psychiatry. 20, 602-8.

Craddock, N. \& Owen, M. J. (2010). The Kraepelinian dichotomy - going, going... but still not gone. Br J Psychiatry 196, 92-5.

Di, Forti.M., Marconi, A., Carra, E., Fraietta, S., Trotta, A., Bonomo, M., Bianconi, F., Gardner-Sood, P., O'Connor, J., Russo, M., Stilo, S.A., Marques, T.R., Mondelli, V., Dazzan, P., Pariante, C., David, A.S., Gaughran, F., Atakan, Z., Iyegbe, C., Powell, J., Morgan, C., Lynskey, M., Murray, R.M., 2015. Proportion of patients in south London with first-episode psychosis attributable to use of high potency cannabis: a case-control study. Lancet Psychiatry 2(3), 233-238.

Droste, S. K., Gesing, A., Ulbricht, S., Muller, M. B., Linthorst, A. C. \& Reul, J. M. (2003). Effects of long-term voluntary exercise on the mouse hypothalamic-pituitary-adrenocortical axis. Endocrinology 144, 3012-23.

Firth, J., Cotter, J., Elliott, R., French, P. \& Yung, A. R. (2015). A systematic review and meta-analysis of exercise interventions in schizophrenia patients. Psychol. Med, 1-19.

Fernandes, B. S., Molendijk, M. L., Kohler, C. A., Soares, J. C., Leite, C. M., MachadoVieira, R., Ribeiro, T. L., Silva, J. C., Sales, P. M., Quevedo, J., Oertel-Knochel, V., Vieta, E., Gonzalez-Pinto, A., Berk, M. \& Carvalho, A. F. (2015). Peripheral brain-derived neurotrophic factor (BDNF) as a biomarker in bipolar disorder: a meta-analysis of 52 studies. BMC Med 13, 289.

Fernandes, B. S., Steiner, J., Berk, M., Molendijk, M. L., Gonzalez-Pinto, A., Turck, C. W., Nardin, P. \& Goncalves, C. A. (2015). Peripheral brain-derived neurotrophic factor in schizophrenia and the role of antipsychotics: meta-analysis and implications. Mol. Psychiatry. 20,1108-19.

Green, J. G., McLaughlin, K. A., Berglund, P. A., Gruber, M. J., Sampson, N. A., Zaslavsky, A. M. \& Kessler, R. C. (2010). Childhood adversities and adult psychiatric disorders in the national comorbidity survey replication I: associations with first onset of DSM-IV disorders. Arch Gen Psychiatry 67, 113-23. 
Kauer-Sant'Anna, M., Kapczinski, F., Andreazza, A.C., Bond, D.J., Lam, R.W., Young, L.T., Yatham, L.N., 2009. Brain-derived neurotrophic factor and inflammatory markers in patients with early- vs. late-stage bipolar disorder. The international journal of neuropsychopharmacology 12(4), 447-458.

Lichtenstein, P., Yip, B. H., Bjork, C., Pawitan, Y., Cannon, T. D., Sullivan, P. F. \& Hultman, C. M. (2009). Common genetic determinants of schizophrenia and bipolar disorder in Swedish families: a population-based study. Lancet 373, 234-239.

Magalhaes, P. V., Dodd, S., Nierenberg, A. A. \& Berk, M. (2012). Cumulative morbidity and prognostic staging of illness in the Systematic Treatment Enhancement Program for Bipolar Disorder (STEP-BD). Aust N Z J Psychiatry 46, 1058-67.

McGorry, P., Keshavan, M., Goldstone, S., Amminger, P., Allott, K., Berk, M., Lavoie, S., Pantelis, C., Yung, A., Wood, S. \& Hickie, I. (2014). Biomarkers and clinical staging in psychiatry. World Psychiatry 13, 211-23.

Mondelli, V., Cattaneo, A., Belvederi, M. M., Di, F. M., Handley, R., Hepgul, N., Miorelli, A., Navari, S., Papadopoulos, A. S., Aitchison, K. J., Morgan, C., Murray, R. M., Dazzan, P. \& Pariante, C. M. (2011). Stress and inflammation reduce brain-derived neurotrophic factor expression in first-episode psychosis: a pathway to smaller hippocampal volume. J Clin Psychiatry 72, 1677-1684.

Nemeroff, C. B. (2016). Paradise Lost: The Neurobiological and Clinical Consequences of Child Abuse and Neglect. Neuron 89, 892-909.

Nuernberg, G. L., Aguiar, B., Bristot, G., Fleck, M. P. \& Rocha, N. S. (2016). Brain-derived neurotrophic factor increase during treatment in severe mental illness inpatients. Transl Psychiatry 6, e985.

Ortiz, B. B., Eden, F. D., de Souza, A. S., Teciano, C. A., de Lima, D. M., Noto, C., Higuchi, C. H., Cogo-Moreira, H., Bressan, R. A. \& Gadelha, A. (2017). New evidence in support of staging approaches in schizophrenia: Differences in clinical profiles between first episode, early stage, and late stage. Compr Psychiatry 73, 93-96.

Scott, J., Leboyer, M., Hickie, I., Berk, M., Kapczinski, F., Frank, E., Kupfer, D. \& McGorry, P. (2013). Clinical staging in psychiatry: a cross-cutting model of diagnosis with heuristic and practical value. Br J Psychiatry 202, 243-5.

Solati, Z., Jazayeri, S., Tehrani-Doost, M., Mahmoodianfard, S., Gohari, M.R., 2015. Zinc monotherapy increases serum brain-derived neurotrophic factor (BDNF) levels and decreases depressive symptoms in overweight or obese subjects: a double-blind, randomized, placebocontrolled trial. Nutritional neuroscience 18(4), 162-168.

Taliaz, D., Stall, N., Dar, D. E. \& Zangen, A. (2010). Knockdown of brain-derived neurotrophic factor in specific brain sites precipitates behaviors associated with depression and reduces neurogenesis. Mol Psychiatry 15, 80-92. 
Yung, A. R., Cotter, J., Wood, S. J., McGorry, P., Thompson, A. D., Nelson, B. \& Lin, A. (2015). Childhood maltreatment and transition to psychotic disorder independently predict long-term functioning in young people at ultra-high risk for psychosis. Psychol. Med, 1-13.

Varese, F., Smeets, F., Drukker, M., Lieverse, R., Lataster, T., Viechtbauer, W., Read, J., van, O. J. \& Bentall, R. P. (2012). Childhood adversities increase the risk of psychosis: a meta-analysis of patient-control, prospective- and cross-sectional cohort studies. Schizophr. Bull 38, 661-671.

Whiteford, H. A., Degenhardt, L., Rehm, J., Baxter, A. J., Ferrari, A. J., Erskine, H. E., Charlson, F. J., Norman, R. E., Flaxman, A. D., Johns, N., Burstein, R., Murray, C. J. \& Vos, T. (2013). Global burden of disease attributable to mental and substance use disorders: findings from the Global Burden of Disease Study 2010. Lancet 382, 1575-1586.

Williams, L. M., Debattista, C., Duchemin, A. M., Schatzberg, A. F. \& Nemeroff, C. B. (2016). Childhood trauma predicts antidepressant response in adults with major depression: data from the randomized international study to predict optimized treatment for depression. Transl Psychiatry 6, e799.

Widom, C. S., Dutton, M. A., Czaja, S. J. \& DuMont, K. A. (2005). Development and validation of a new instrument to assess lifetime trauma and victimization history. J Trauma Stress 18, 519-31. 
Table 1. Total sample sociodemographic and clinical characteristics

\begin{tabular}{|l|l|l|l|l|l|}
\hline Variables & $\begin{array}{l}\mathrm{BD} \\
(\mathrm{n}=254)\end{array}$ & $\begin{array}{l}\mathrm{SZ} \\
(\mathrm{n}=589)\end{array}$ & $\begin{array}{l}\text { Controls } \\
(\mathrm{n}=603)\end{array}$ & $\mathrm{F} / \mathrm{X}^{2}$ & $\mathrm{p}$ \\
\hline Sex, M n (\%) & $99(39.8)$ & $349(59.9)$ & $317(51.8)$ & $\mathrm{X}^{2}=29.8$ & $<0.001$ \\
\hline Age, years mean \pm SD & $33.5 \pm 11.7$ & $30.0 \pm 9.8$ & $32.8 \pm 9.6$ & $\mathrm{~F}=16.1$ & $<0.001$ \\
\hline Caucasians n (\%) & $226(89.0)$ & $463(78.6)$ & $588(96.1)$ & $\mathrm{X}^{2}=109.9$ & $<0.001$ \\
\hline Psychotic episodes, mean \pm SD & $1.3 \pm 2.5$ & $2.4 \pm 3.7$ & & $\mathrm{~F}=10.9$ & 0.001 \\
\hline Manic episodes, mean \pm SD & $1.7 \pm 2.8$ & $0.3 \pm 1.4$ & & $\mathrm{~F}=91.8$ & $<0.001$ \\
\hline Depressive episodes, mean \pm SD & $5.8 \pm 8.0$ & $2.0 \pm 5.9$ & & $\mathrm{~F}=54.5$ & $<0.001$ \\
\hline BDNF plasma, mean \pm SD* & $5.7 \pm 3.3$ & $6.0 \pm 3.8$ & $6.6 \pm 3.6$ & $\mathrm{~F}=7.9$ & $<0.001$ \\
\hline Daily smoking, yes (\%) & $139(54.7)$ & $316(54.4)$ & & $\mathrm{X}^{2}<0.01$ & 0.93 \\
\hline
\end{tabular}

3 missing data on ethnicity. Ethnicity,” other” consists of 32 Africans, 9 Hispanics, 2 Same, 64 Asians, 29 Arabic, mixed or other=30. Log values of BDNF. Mean $\pm S D, B D=1.8 \pm 0.5$; $S Z=1.8 \pm 0.5$; Controls $=1.9 \pm 0.5$. 
Table 2: Number of episodes and BDNF levels

\begin{tabular}{lcccc} 
Variable & se & $\beta$ & $\mathrm{t}$ & $\mathrm{p}$ \\
\hline $\begin{array}{l}\text { Psychotic } \\
\text { episodes* }\end{array}$ & 0.008 & 0.06 & 1.40 & 0.16 \\
$\begin{array}{l}\text { Depressive } \\
\begin{array}{l}\text { episodes* } \\
\text { Manic episodes* }\end{array}\end{array}$ & 0.003 & -0.11 & -2.07 & $\mathbf{0 . 0 4}$ \\
\hline Ad & 0.01 & 0.09 & 1.04 & 0.30
\end{tabular}

Adjusted; group, years since blood sampling, age, sex, age at onset, and daily smoking (yes/no) *=number of episodes 
Table 3: Childhood trauma is associated with lower BDNF plasma in the total sample and stratified into females and in males

\begin{tabular}{llll}
\hline & Total sample & Females only & Males only \\
\hline Total trauma & $r=-0.16$ & $r=-0.22$ & $r=-0.11$ \\
CTQ & $P<0.001$ & $P<0.001$ & $P=0.047$ \\
\hline Sexual abuse & $r=-0.16$ & $r=-0.21$ & $r=-0.09$ \\
& $P<0.001$ & $P<0.001$ & $P=$ n.s. \\
\hline Physical abuse & $r=-0.14$ & $r=-0.012$ & $r=-0.16$ \\
& $P<0.001$ & $P<0.05$ & $P<0.05$ \\
\hline Emotional abuse & $r=-0.14$ & $r=-0.20$ & $r=-0.07$ \\
& $P<0.001$ & $P<0.001$ & $P=$ n.s. \\
\hline Emotional neglect & $r=-0.13$ & $r=-0.20$ & $r=-0.07$ \\
& $P \leq 0.01$ & $P<0.001$ & $P=$ n.s. \\
\hline Physical neglect & $r=-0.08$ & $r=-0.11$ & $r=-0.05$ \\
& $P<0.05$ & $P=$ n.s. & $P=$ n.s. \\
\hline
\end{tabular}

Spearman's correlation. BDNF=Brain-Derived Neurotrophic Factor, plasma levels. CTQ=Childhood Trauma Questionnaire. 
Figure 1: BDNF Levels and diagnoses

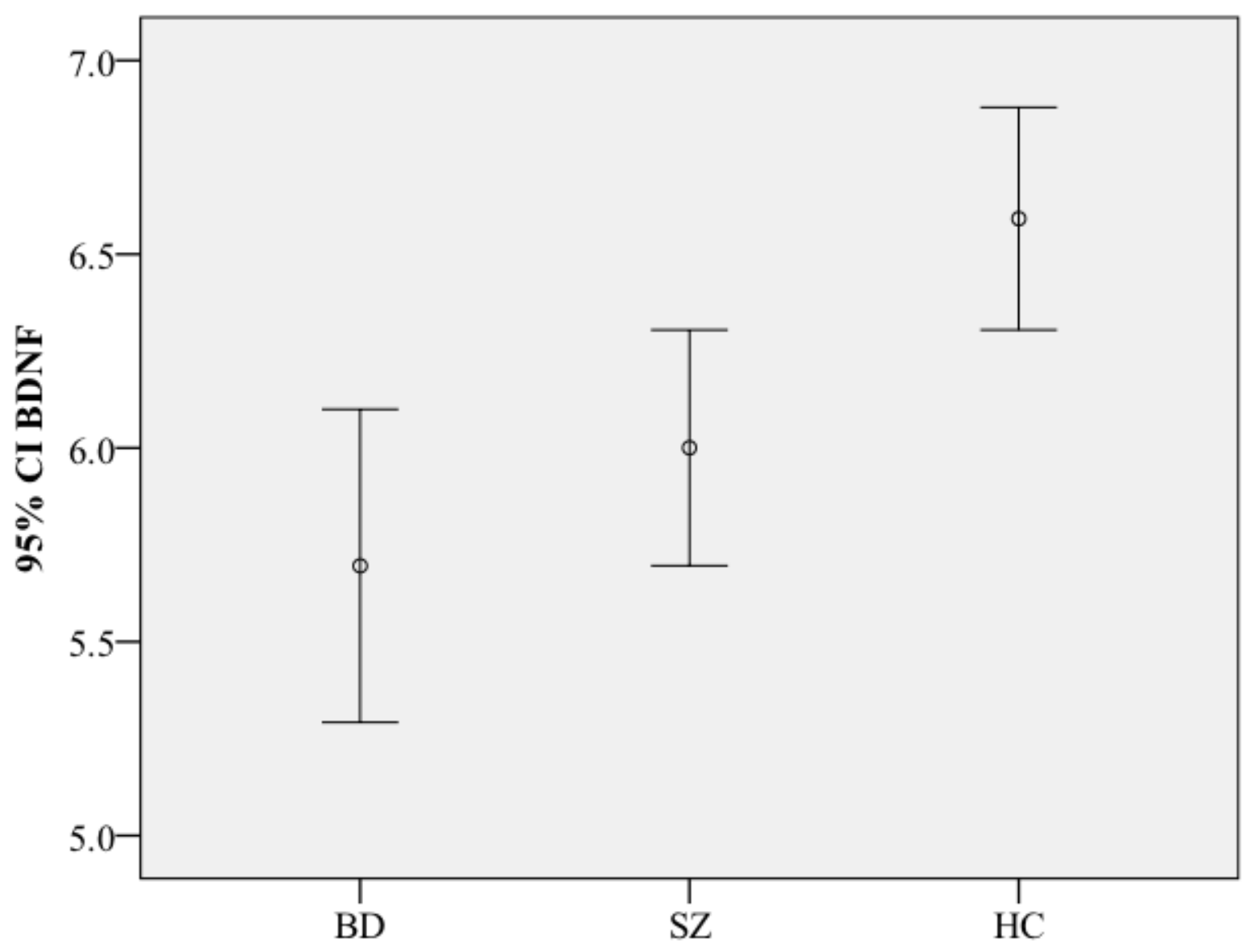

ANOVA, f=7.95, $\mathrm{p}<0.001$. BD, N=254; SZ, N=589, HC, N=603. Controls $>$ SZ, BD. No statistical significant differences in BDNF levels within the patients group. $\mathrm{BD}=$ bipolar disorder, SZ=schizophrenia, and $\mathrm{HC}=$ healthy controls. 
Figure 2: Longer time in remission of affective symptoms is associated with higher BDNF levels

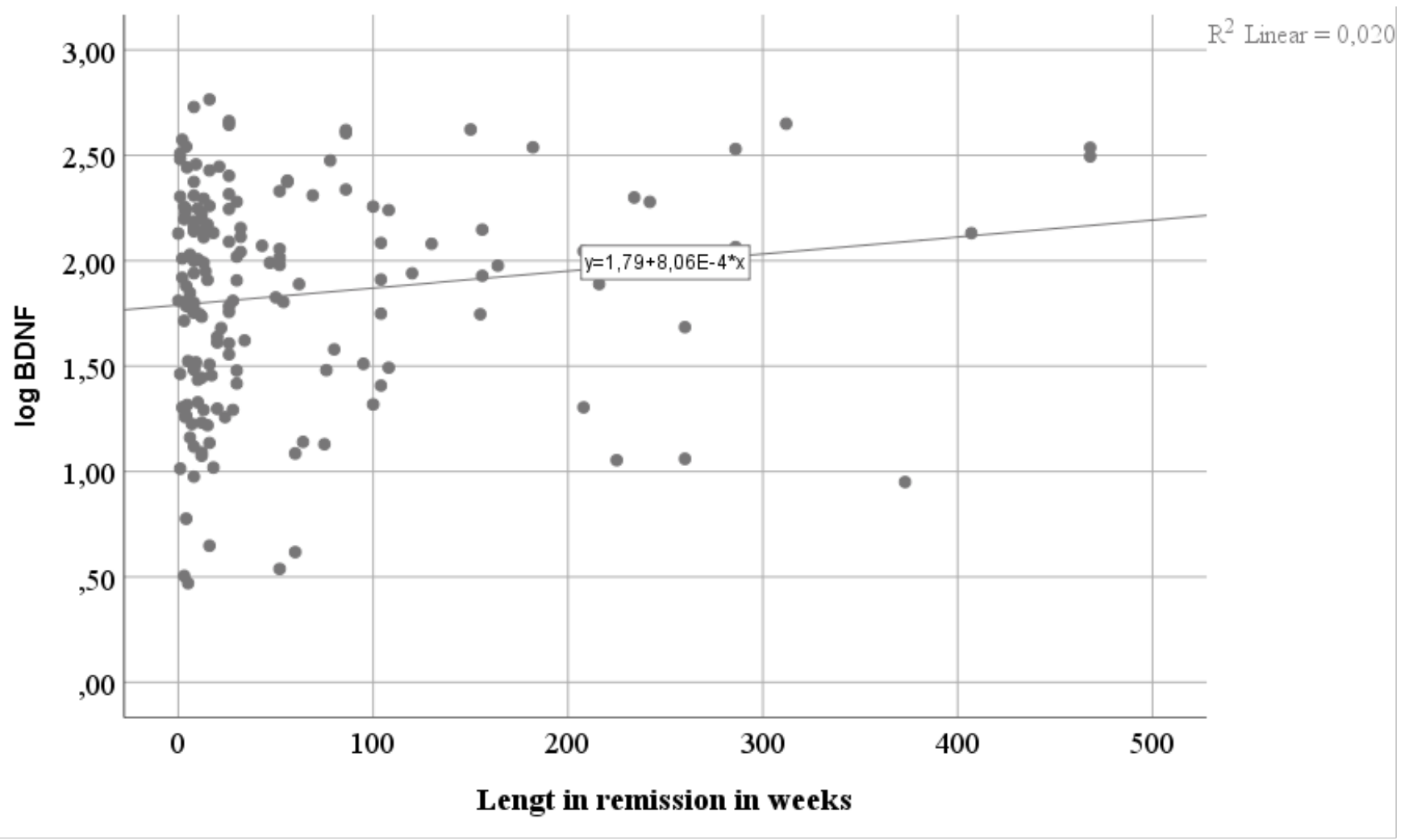

Regression analyses, $\beta=0.19, \mathrm{p}=0.02$ 


\section{Supplementary Material}

Table S1: Childhood trauma stratified into groups

\begin{tabular}{|l|l|l|l|l|l|}
\hline & SZ & BD & HC & Statistics & Posthoc \\
\hline $\begin{array}{l}\text { CTQ total score, } \\
\text { mean } \pm \text { SD }\end{array}$ & $46.29 \pm 15.80$ & $42.32 \pm 15.54$ & $30.00 \pm 5.39$ & $\begin{array}{l}\mathrm{F}=103.28, \mathrm{df}=2, \\
P<0.001\end{array}$ & $\mathrm{HC}<\mathrm{SZ}, \mathrm{BD}$ \\
\hline $\begin{array}{l}\text { Sexual abuse, } \\
\text { mean } \pm \text { SD }\end{array}$ & $6.80 \pm 3.46$ & $6.89 \pm 4.09$ & $5.13 \pm 0.65$ & $\begin{array}{l}\mathrm{F}=24.73, \mathrm{df}=2, \\
P<0.001\end{array}$ & $\mathrm{HC}<\mathrm{SZ}, \mathrm{BD}$ \\
\hline $\begin{array}{l}\text { Physical abuse, } \\
\text { mean } \pm \text { SD }\end{array}$ & $7.00 \pm 3.28$ & $6.59 \pm 3.46$ & $5.15 \pm 0.64$ & $\begin{array}{l}\mathrm{F}=35.29, \mathrm{df}=2, \\
P<0.001\end{array}$ & $\mathrm{HC}<\mathrm{SZ}, \mathrm{BD}$ \\
\hline $\begin{array}{l}\text { Emotional abuse, } \\
\text { mean } \pm \text { SD }\end{array}$ & $11.26 \pm 5.18$ & $10.19 \pm 4.65$ & $6.23 \pm 2.06$ & $\begin{array}{l}\mathrm{F}=77.24, \mathrm{df}=2, \\
P<0.001\end{array}$ & $\mathrm{HC}<\mathrm{SZ}, \mathrm{BD}$ \\
\hline $\begin{array}{l}\text { Emotional neglect, } \\
\text { mean } \pm \text { SD }\end{array}$ & $12.64 \pm 5.01$ & $11.79 \pm 4.93$ & $7.36 \pm 2.98$ & $\begin{array}{l}\mathrm{F}=73.76, \mathrm{df}=2, \\
P<0.001\end{array}$ & $\mathrm{HC}<\mathrm{SZ}, \mathrm{BD}$ \\
\hline $\begin{array}{l}\text { Physical neglect, } \\
\text { mean } \pm \text { SD }\end{array}$ & $8.27 \pm 3.13$ & $7.19 \pm 2.90$ & $5.88 \pm 1.61$ & $\begin{array}{l}\mathrm{F}=44.42, \mathrm{df}=2, \\
P<0.001\end{array}$ & $\begin{array}{l}\mathrm{HC}<\mathrm{SZ}, \mathrm{BD} \\
\mathrm{BD}<\mathrm{SZ}\end{array}$ \\
\hline
\end{tabular}

CTQ= Childhood Trauma Questionnaire. SZ= schizophrenia, BD= Bipolar Disorder; HC= healthy controls. As the CTQ variables were skewed, ANOVA reflects log transferred values.

Table S2: 1 year follow-up and BDNF levels (N=91)

\begin{tabular}{lll}
\hline & BDNF levels & BDNF change \\
& 1 year follow-up & $\begin{array}{l}\text { from baseline to } \\
1 \text { year follow-up }\end{array}$ \\
\hline Remission baseline & n.s. & n.s. \\
Remission 1 year follow-up, & n.s. & n.s. \\
GAF, baseline & n.s. & n.s. \\
GAF, 1 year follow-up & n.s. & n.s. \\
PANSS, baseline & n.s. & n.s. \\
PANSS, year follow-up & n.s. & n.s. \\
\hline
\end{tabular}

Analyses adjusted for sex, age, cigarette smoking and time of stored samples.GAF=Global Assessment of Functioning Scale, PANSS=Positive and Negative Syndrome scale. BDNF= Brain-Derived Neurotrophic Factor. n.s.= Not statistically significant. 
Figure S1: More childhood trauma subtypes were associated with lower BDNF in the total sample.

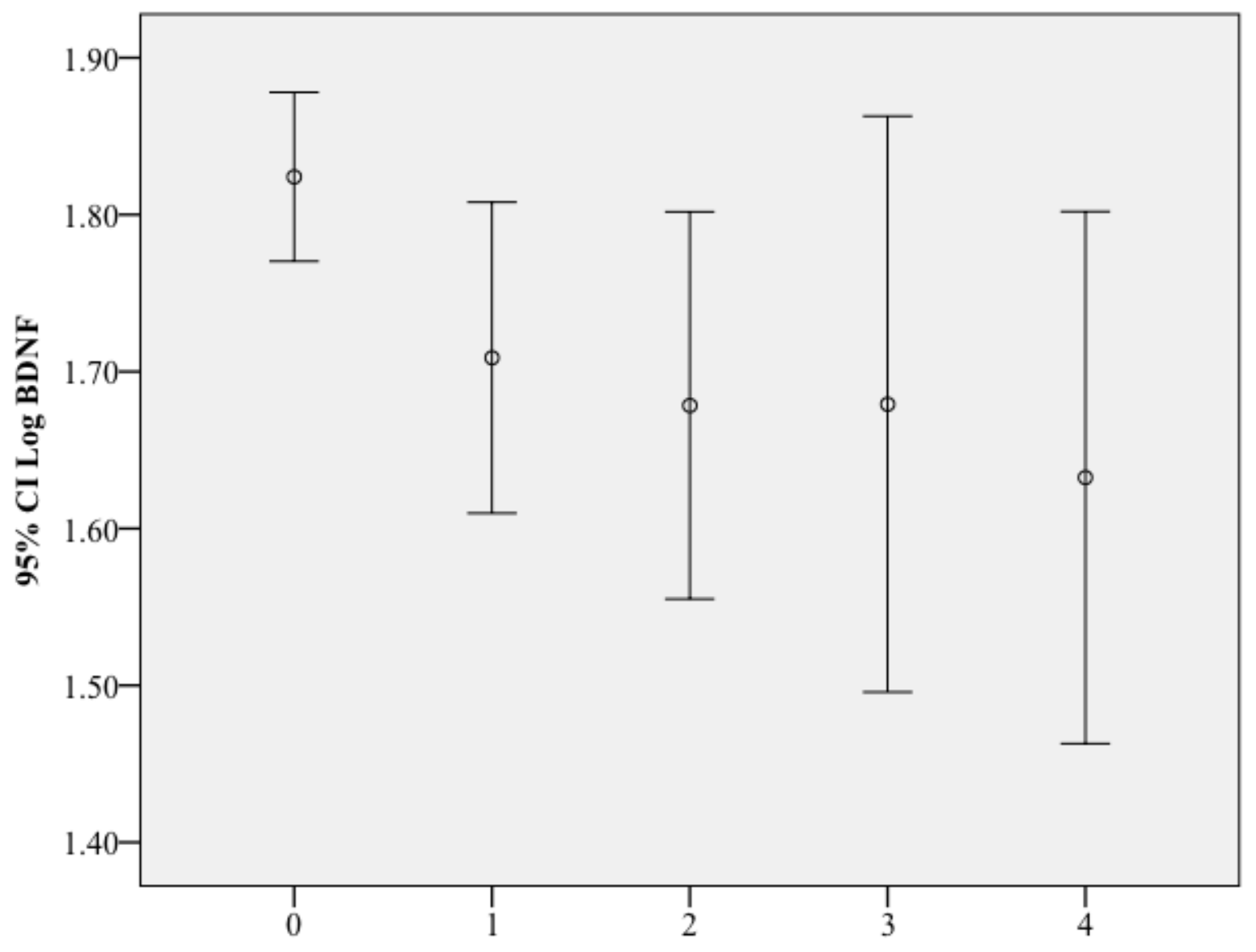

$\mathrm{F}=3.09, \mathrm{P}=0.02$. $0=$ no trauma, $\mathrm{N}=386,1=$ one type of childhood trauma, $\mathrm{N}=101 ; 2=$ two types of childhood trauma, $\mathrm{N}=57 ; 3$ types of trauma, $\mathrm{N}=35$, and 4 or more types of trauma, $\mathrm{N}=46$. 
Figure S2: Patients reporting sexual abuse had lower BDNF levels than patients who did not report sexual abuse

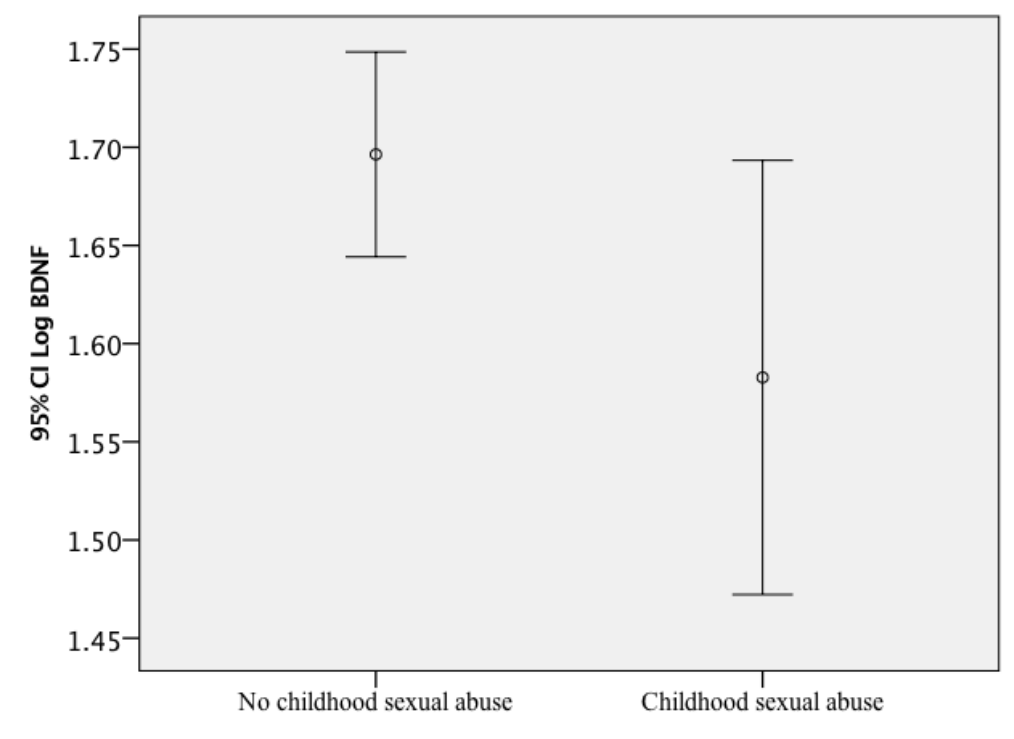

$\mathrm{F}=3.08$, $\mathrm{p}=0.049$. "No childhood sexual abuse", $\mathrm{N}=337 ; 1=$ "Childhood sexual abuse", $\mathrm{N}=88$. Childhood sexual abuse was defined as having moderate to severe score on the childhood sexual abuse domain from the CTQ (Bernstein et al., 1994). 
Figure S3: Patients reporting sexual abuse had more depressive episodes

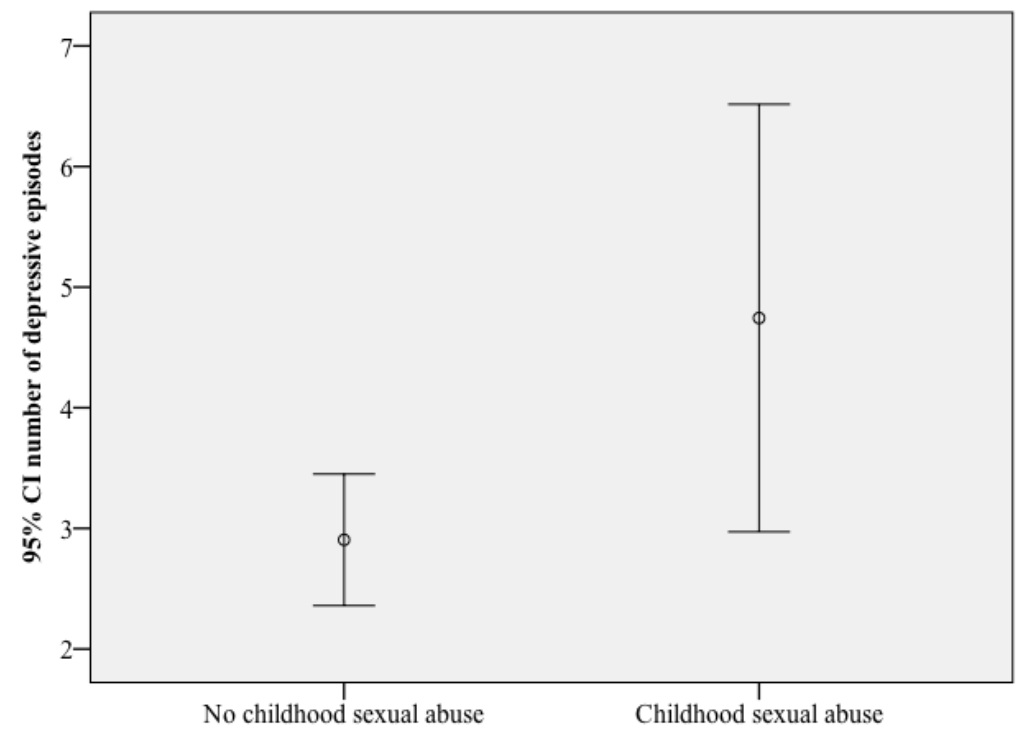

$\mathrm{F}=6.69$, $\mathrm{p}=0.01$. "No childhood sexual abuse”, N=326; "childhood sexual abuse”, N=82. Childhood sexual abuse was defined as having moderate to severe score on the childhood sexual abuse domain from the CTQ (Bernstein et al., 1994). 
Figure S4: Patients with childhood sexual abuse reported more psychotic episodes

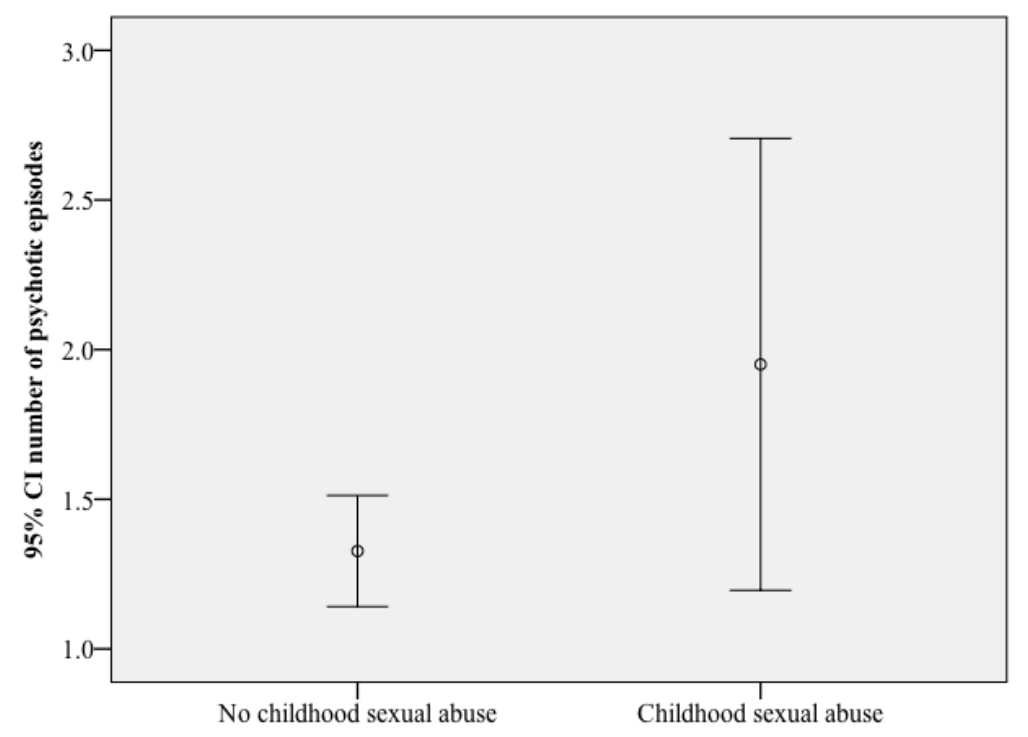

$\mathrm{F}=5.41$, $\mathrm{p}=0.02$. "No childhood sexual abuse", $\mathrm{N}=318$; "childhood sexual abuse”, $\mathrm{N}=81$. Childhood sexual abuse was defined as having moderate to severe score on the childhood sexual abuse domain from the CTQ (Bernstein et al., 1994). 